Oral (003)

Epithelial Ovarian Cancer including Borderline Tumor

https://doi.org/10.3802/jgo.2021.32.51.003

\section{Effect of cytoreductive surgery and hyperthermic intraperitoneal chemotherapy on epithelial ovarian, fallopian tube and peritoneal cancer: an institutional review of outcomes and its clinical implications}

Romelyn Imperio-Onglao, J Jericho Thaddeus P. Luna

UP-Philippine General Hospital, Manila, Philippines (rapimperio@gmail.com)

Objective: The study aimed to determine the efficacy and safety of cytoreductive surgery (CRS) with hyperthermic intraperitoneal chemotherapy (HIPEC) vs. CRS for patients with epithelial ovarian, fallopian tube and peritoneal cancer.

Methods: This retrospective cohort study included 50 patients (20 CRS with HIPEC, and 30 patients who underwent CRS). Records of these patients from 2014-2020 were reviewed and tabulated.

Results: Recurrence rate between CRS with HIPEC and CRS alone was not statistically significant ( $50 \%$ vs. $43 \%$, $p=0.774)$. Median time to recurrence was 10 and 9 months ( $p=0.636$ ). Five percent expired in the HIPEC group, and 13\% expired in the CRS ( $\mathrm{p}=0.636)$. More post-operative complications were noted in the HIPEC group ( $\mathrm{p}=0.007$ ), but only 2 cases had grade 3 to 4 complications $(10 \%)$. HIPEC patients had longer operative time $(\mathrm{p}<0.001)$ and post-operative hospital stay $(\mathrm{p}=0.026)$. There were no intra-or peri-operative mortalities in both groups. Conclusion: CRS with HIPEC and CRS alone showed similar time to recurrence and recurrence rate. CRS with HIPEC had low risk of grade 3-4 complications and may still be considered as a treatment option for advanced and recurrent epithelial ovarian, fallopian tube and peritoneal cancer.

Oral (004)

Surgical Techniques Perioperative Management

https://doi.org/10.3802/jgo.2021.32.\$1.004

\section{Cytoreductive surgery after neoadjuvant chemotherapy in ovarian cancer: Does Neoadjuvant chemotherapy really ease the burden?}

\section{Mukurdipi Ray," Talluri Sri Harsha Vardhan Surya, Ashutosh Mishra \\ All India Institute of Medical Sciences, New Delhi, India (major.mdray@gmail.com)}

Objective: Neoadjuvant chemotherapy (NACT) is used in most advanced ovarian cancer cases, not amenable to upfront surgery.
Apart from the anesthetic implications of chemotherapy, the desmoplastic reaction to chemotherapy also poses a difficulty to surgical resection compared to upfront surgery. Interval cytoreductive surgery (CRS) requires surgical expertise, knowledge about the disease recurrence pattern after NACT, and diligent perioperative care to minimize complications. The present study describes the standardized surgical technique to reduce complications and increase recurrence free survival (RFS), as practiced by the author routinely.

Methods: An audit of a prospectively maintained computerized database in the department of surgical oncology was done. Intraoperative and immediate postoperative outcomes and patterns of recurrence and RFS were analyzed. We described our surgical technique perfected over 106 cases of post-NACT, CRS and hyperthermic intraperitoneal chemotherapy (HIPEC).

Results: In 516 cases of ovarian cancer operated from January 2014 to November 2020, CRS and HIPEC was performed after NACT in 106 patients. Nerve-sparing hysterectomy and nerve-sparing retroperitoneal lymph node dissection were performed in 33\% (31/106) cases. The mean duration of surgery was 310.8 minutes. The mean duration of hospital stay was 6.82 days, and the mean intensive care unit stay duration was 1.7 days. The predominant pattern of recurrence was peritoneal, followed by nodal recurrence. Conclusion: Interval CRS + HIPEC is a unique challenge with increased surgical complexity. Standardization of the surgical technique can reduce associated complications and optimizes outcomes.

Oral (005)

Epithelial Ovarian Cancer including Borderline Tumor https://doi.org/10.3802/jgo.2021.32.S1.005

\section{Effect of BRCA mutational status on survival outcomes after secondary cytoreduction in platinum-sensitive relapsed ovarian cancer}

Se Ik Kim, Junhwan Kim, Jae-Weon Kim, Maria Lee*

Seoul National University College of Medicine, Seoul, Korea (marialeemd@gmail.com)

Objective: To investigate impact of BRCA1/2 mutational status on survival outcomes after secondary cytoreduction in platinumsensitive relapsed (PSR), epithelial ovarian cancer (EOC). Methods: We identified patients who underwent secondary cytoreductive surgery for PSR EOC between January 2009 and March 2021 and who received BRCA1/2 gene testing by either germline or somatic methods. Patients were divided into BRCA mutation and wild-type groups, followed by comparisons of clinicopathologic characteristics and survival outcomes after secondary-line treatment. 
Results: In total, 22 and 53 patients were assigned to BRCA mutation and wild-type groups, respectively. Between the 2 groups, no differences in patient age, proportions of highgrade serous carcinoma and bevacizumab users, and the use of maintenance therapy were observed. However, the BRCA mutation group had a significantly longer platinum-free interval ( $\mathrm{p}=0.021)$ and a higher rate of complete cytoreduction with borderline statistical significance ( $95.5 \%$ vs. $73.6 \%$; $\mathrm{p}=0.053)$, compared to the BRCA wild-type group. Of 22 patients in the BRCA mutation group, 8 (36.4\%) received PARP inhibitor maintenance therapy after completion of second-line platinum-based combination chemotherapy. After a median follow-up of 38.2 months, the groups showed similar overall survival; however, the BRCA mutation group displayed better progression-free survival (PFS; median, 28.4 vs. 18.4 months; $\mathrm{p}=0.012$ ). Multivariate analyses identified BRCA1/2 mutation as an independent favorable prognostic factor for PFS (adjusted hazard ratio $=0.419 ; 95 \%$ confidence interval $=0.218-0.806$; $\mathrm{p}=0.009)$. Consistent results were observed among 60 patients who achieved complete cytoreduction.

Conclusion: In the case of secondary cytoreductive surgery for PSR EOC, patients with BRCA1/2 mutations have a better prognosis with longer PFS than those lacking BRCA mutations. Further studies with a large sample size are warranted to confirm our findings.

Oral (006)

Epithelial Ovarian Cancer including Borderline Tumor

https://doi.org/10.3802/jgo.2021.32.S1.006

\section{Clinical characteristics of early-stage clear cell ovarian cancer: a 10-year retrospective experience in Ren Ji Hospital}

\section{Xiaoshi Liu, Wen $\mathrm{Di}^{*}$}

Ren Ji Hospital, School of Medicine, Shanghai Jiao Tong University, Shanghai, China (diwen163@163.com)

Objective: Ovarian clear cell carcinoma (OCCC) is a distinct subtype of epithelial ovarian cancer with unique clinical and molecular characteristics. It is likely to be found at an earlystage and its prognosis is relatively good. However, it has recently been reported that in early-stage OCCC, some recur more frequently and become chemoresistant according to subtypes, due to occult metastasis. This study was conducted to learn more about the clinical characteristics and outcomes of early-stage OCCC, as well as to provide more information to help with clinical diagnosis and therapy.

Methods: The clinical features such as age, preoperative cancer antigen 125 (CA125), CA19-9, platelet, plasma D-dimer, systemic inflammatory response (SIR) markers, treatment and survival outcomes of 70 patients with International Federation of Gynecology and Obstetrics I-II OCCC treated at our center between August 2010 and April 2020 were investigated in this retrospective analysis. Initially, receiver operating characteristic curve analysis for overall survival (OS) was used to determine optimal cut-off values for preoperative parameters $(\mathrm{CA} 125=52.65$, CA19-9 $=21.3$, platelet $=223.5$, SIR index [SIRI]=1.289). Kaplan-Meier curves were used for the analysis of OS and progression-free survival (PFS). The p-values were calculated by a log-rank test. Multivariate Coxregression analysis was used to further assess the variables proved significant in univariate analysis. $\mathrm{p}<0.05$ was considered statistically significant.

Results: Overall, the mean age of participants was $57.46 \pm 9.41$ years old. All patients underwent hysterectomy with bilateral salpingo-oophorectomy, and $87.1 \%$ of them underwent lymphadenectomy. Of the 62 women with available chemotherapy data, $42(67.7 \%)$ received one cycle intraperitoneal chemotherapy with cisplatin and 59 (95.2\%) underwent more than 3 cycles of chemotherapy. The 48 patients received open procedures and the rest underwent laparoscopy. There were 14 recurrences and 3 deaths during the median follow-up duration of 55.2 months. The median CA125 level was 56.27 IU/mL (range 5.81-602.8 IU/mL), with normal CA125 levels seen in 20 patients. Almost three quarters of patients (77.1\%) assayed had a normal CA19-9, with a median level of 15.6 IU/mL. In this study, no statistically significant correlations between the differential pretreatment levels of CA125 $(\mathrm{p}=0.87$ for OS, $\mathrm{p}=0.71$ for PFS)/CA19-9 $(\mathrm{p}=0.05$ for OS, $\mathrm{p}=0.95$ for PFS) and clinical outcomes in patients with early-stage OCCC were found. SIRI $(p=0.008)$ and platelet $(p=0.002)$ levels were identified as prognostic factors for OS. SIRI $(p=0.024)$ was also prognostic factor for PFS. Patients who received one cycle intraperitoneal chemotherapy were related to an obviously better prognosis ( $\mathrm{p}=0.032$ for OS, $\mathrm{p}=0.048$ for PFS). Furthermore, multivariate analysis showed that an increase in SIRI is a significant independent prognostic factor for poor prognosis (hazard ratio=3.192, $\mathrm{p}=0.00215$ ).

Conclusion: Early-stage OCCC often presents with mildly elevated CA125 and adding one cycle intraperitoneal chemotherapy can offer additional survival benefit to patients. Pretreatment evaluated SIRI might be potential biomarkers for worse response to first-line chemotherapy and poorer clinical outcomes. Therefore, more specific treatment strategies and a rigorous follow-up plan can be formulated for patients with early-stage OCCC based on clinical characteristics and preoperative parameters. 